\title{
Influence of Protein Supplement on the Process of Rehabilitation in Damage of Muscles Caused by Physical Loads
}

\author{
Alexander Plakida ${ }^{1, ~}$, Igor Bondarev ${ }^{2}$, Sergey Gushcha $^{3}$ \\ ${ }^{1}$ Department of Physical Rehabilitation, Sports Medicine, Physical Training and Valeology, Odessa National Medical University, Odessa, \\ Ukraine \\ ${ }^{2}$ Department of Physical Rehabilitation, SE Ukrainian Research Institute of Medical Rehabilitation and Balneology Ministry of Health of \\ Ukraine, Odessa, Ukraine \\ ${ }^{3}$ Department of Basic Research, SE Ukrainian Research Institute of Medical Rehabilitation and Balneology Ministry of Health of Ukraine, \\ Odessa, Ukraine
}

Email address:

aplakida@mail.ru (A. Plakida)

${ }^{*}$ Corresponding author

To cite this article:

Alexander Plakida, Igor Bondarev, Sergey Gushcha. Influence of Protein Supplement on the Process of Rehabilitation in Damage of Muscles Caused by Physical Loads. European Journal of Clinical and Biomedical Sciences. Vol. 3, No. 6, 2017, pp. 134-138.

doi: $10.11648 /$ j.ejcbs.20170306.16

Received: October 31, 2017; Accepted: December 14, 2017; Published: December 27, 2017

\begin{abstract}
Resistance exercise is a common mode of training may precipitate temporary exercise-induced muscle damage that manifests as a reduction in neuromuscular function, reduced range of motion, increased muscle soreness, limb swelling and the elevation of intramuscular proteins in blood. The purpose of this study was to study the effect of protein supplements on the process of rehabilitation for muscle injuries caused by physical stress. The study involved 21 clinically healthy men. Participants perform eccentric contractions of the quadriceps femoris muscle. All participants in the studies were randomly assigned to three groups. The first group received the protein supplement before exercise and placebo after the load. The second group received a placebo before the load and a protein drink after. The third group received a placebo both before and after the load. Before the load and 24, 48, 72 hours after the load, the maximum possible reduction (MPR) of the quadriceps muscle was studied, the severity of muscle soreness (MS), the content of creatine kinase (CK) in the serum. The dynamics of changes in the studied indicators was unidirectional in all groups. The index of the maximum possible reduction reached a minimum value 24 hours after exercise and gradually returned to the initial values in 72 hours. Between the first group and the control group no significant differences were found in any of the stages of restitution. At the same time, there was a significant difference in recovery in the participants of the second group and the control group at 48 hours after the load $(94 \pm 3.81$ and $84 \pm 3.12$, P <0.05), which indicates a faster recovery of contractile ability of the muscles in the studied persons of second group. Just as in the study of MPR dynamics, there were no significant differences between the indices of participants in the first and control groups. In the case of participants in the third group, significant differences in indices are determined in the intervals 48 and 72 hours after the load. The peak values of CK recorded 48 hours after the load. Significant differences were found between second group and control (1448 \pm 208 and $864 \pm 113, \mathrm{P}<0.05)$. Consequently the intake of protein supplements immediately after physical exertion reduces the duration of the subjective sensation of muscle soreness and promotes the fastest regeneration of damaged muscle fibers.
\end{abstract}

Keywords: Physical Load, Protein Supplements, Creatinekinase, Muscle Pain

\section{Introduction}

Resistance exercise is a common mode of training and is considered an integral part in the athletes' training regimen. Although many resistance exercises require both shortening and lengthening contractions, it has been well documented that exercise biased by lengthening contractions are a more 
powerful stimulus for neuromuscular adaptation compared to shortening contractions $[1,16,23]$. As a consequence, many athletes will routinely incorporate this exercise modality in order to maximize the potential adaptations from lengthening contractions. However, lengthening contractions, particularly when high forces are generated, precipitate temporary exercise-induced muscle damage (EIMD) that can last for several days after the initial bout [7]. This EIMD manifests as a reduction in neuromuscular function, reduced range of motion, increased muscle soreness, limb swelling and the elevation of intramuscular proteins in blood [21, 25]. These signs and symptoms impair muscle function and inhibit the potential to engage in high intensity exercise on subsequent days, which is often required by athletic populations.

There are the following basic hypotheses about the nature and nature of painful sensations in the muscles:

Damage or micro-ruptures of muscles;

Damage to the fascial connective tissue;

Accumulation of metabolic products and associated increased osmotic pressure in the muscles;

Excess concentration of lactic acid;

Local spasm of motor units $[6,25]$.

In an attempt to reduce the negative effects of EIMD a number of interventions have been explored; these include cold water immersions [9], antioxidant supplementation, ergogenic aids [4], non-steroidal anti-inflammatory drugs and nutritional interventions [15]. One of the most promising methods of rehabilitation in this case is the use of protein supplements $[5,14,18,22]$. Commercially, the two most popular types of proteins in supplemental form are whey and casein. Recent investigations have detailed the serum amino acid responses to ingesting different protein types. Using amino acid tracer methodology, it was demonstrated that whey protein elicits a sharp, rapid increase of plasma amino acids following ingestion, while the consumption of casein induces a moderate, prolonged increase in plasma amino acids that was sustained over a 7$\mathrm{hr}$ postprandial time period. The differences in the digestibility and absorption of these protein types may indicate that the ingestion of "slow" (casein) and "fast" (whey) proteins differentially mediate whole body protein metabolism due to their digestive properties. Other studies have shown similar differences in the peak plasma levels of amino acids following ingestion of whey and casein fractions (i.e., whey fractions peaking earlier than casein fractions). These examples have shown mixed success, however one nutritional intervention, branched chain amino acids (BCAA), have shown a reasonable degree of efficacy in reducing the effects of EIMD; in the most part following strenuous endurance exercise. BCAA are a group of essential amino acids that are a key substrate for protein synthesis and recovery [5]. Furthermore, BCAA conserve muscle mass in conditions characterized by protein loss and catabolism [4] and a recent review has proposed BCAA to provide a therapeutic effect following damaging resistance exercise [7]. Indeed, studies examining recovery from heavy endurance activity [20] have shown evidence that
BCAA are beneficial in reducing muscle damage and accelerating the recovery process. Whilst this positive evidence is encouraging, muscle damage is far more prevalent following high intensity resistance exercise, although few studies have examined the efficacy of BCAA following damaging resistance exercise. Nosaka et al. [21] showed that amino acid supplementation (containing around $60 \%$ BCAA) was effective in reducing muscle damage and soreness when consumed immediately before and during the four recovery days that followed a damaging bout of lengthening contractions. Additionally, in a recent well-controlled example [10], muscle soreness was reduced with BCAA; however, changes in blood indices or recovery of muscle function were absent. The aforementioned studies $[10,21]$ used untrained volunteers and an isolated muscle group, which are not wholly representative of the stimulus often encountered by many athletic populations who routinely use damaging lengthening-biased resistance exercise as a training stimulus. Shimomura et al. [24] examined BCAA supplementation in untrained females and whilst these authors demonstrated some efficacy in reducing indices of damage in the BCAA group, the placebo control consumed carbohydrate, which has been shown to facilitate protein uptake [5], thus having a synergistic effect to any exogenous protein consumed following the laboratory visit. Interestingly, and in some support of this supposition, Stock et al. [26] showed that in a mixed sex group of trained participants there were no differences in damage indices between a carbohydrate versus a carbohydrate + leucine supplement. This study contradicts the general findings from other research, which may partly be attributable to a methodological difference such as providing leucine alone (and not leucine, isoleucine and valine combined). Additionally, Sharp and Pearson [23] recently examined BCAA supplementation during a resistance training programme designed to induce overreaching. These authors showed some efficacy with BCAA supplementation in resistance-trained individuals (with the exception of creatine kinase), however, the study was not focused on damaging exercise and/or recovery making the findings somewhat disparate. Nevertheless, the current evidence is promising and therefore hypothesized the magnitude of EIMD in resistance-trained individuals would be lower with BCAA supplementation compared to a placebo control. Consequently, the aim of this study was to investigate the effect of BCAA supplementation on recovery from a sport-specific damaging bout of resistance exercise in trained volunteers. In this way number of researchers have shown that the addition of branched-chain amino acids activates the synthesis and reduces the breakdown of the muscle protein $[3,8,12,23,29]$, but the data of other authors do not confirm these findings $[6,7$, $19,21,28]$. In this regard, the purpose of this study was to study the effect of protein supplements (whey protein) on the process of rehabilitation for muscle injuries caused by physical stress. 


\section{Methods of Research}

\subsection{Participants}

The study involved clinically healthy men aged 18-25 years, 21 people. The average weight of the subjects was $76.3 \pm 8.6 \mathrm{~kg}$, the average height was $179.6 \pm 5.3 \mathrm{~cm}$. Over the past three months before the research, none of them were engaged in regular sports training.

\subsection{Experimental Design}

The design of the double-blind study was as follows. Participants were asked to perform 50 eccentric contractions of the quadriceps femoris muscle for $60 \mathrm{sec}$ on the Cybex Humac Norm isokinetic dynamometer. Fifteen minutes before the exercise, the subjects studied for two minutes drank the first drink. After this, a test with physical activity was performed and, immediately after it termination, the subjects drank a second drink.

All participants in the studies were randomly assigned to three groups of 7 people each. The first group (Pre) received the protein supplement before exercise and placebo after the load. The second group (Post) received a placebo before the load and a protein drink after. The third group (Control) received a placebo both before and after the load.

The protein drink included $23 \mathrm{~g}$ of whey protein and $75 \mathrm{~g}$ of carbohydrates $(392 \mathrm{kcal})$ mixed with $300 \mathrm{ml}$ of water. Placebo consisted of 75 grams of carbohydrates mixed with $300 \mathrm{ml}$ of water.

The subjects were not aware of what beverages they consume before and after the load.

Before the load and 24, 48, 72 hours after the load, the maximum possible reduction (MPR) of the quadriceps muscle was studied, the severity of muscle soreness (MS), the content of creatine kinase (CK) in the serum. MPR was assessed by the maximum possible mechanical effort with extension of the quadriceps femoris muscle $[15,17]$. The value of MPR before the load was taken as $100 \%$ and further results were calculated as a percentage of the initial level. The MS was determined by the following method. Participants were asked to perform and hold the squat position (angle of the knee $90^{\circ}$ ), and at that moment to estimate the level of sensation of muscular pain on a $200 \mathrm{~mm}$ visual analogue scale $[10,15,16]$. The scale consisted of a line from $0 \mathrm{~mm}$ (without pain) to $200 \mathrm{~mm}$ (unbearably painful). The concentration of $\mathrm{CK}$, as a universally recognized marker of muscle damage, was determined by the Warburg spectrophotometric test [2].

The data obtained were processed using variation statistics methods of the XLSTAT statistical package.

\section{Results and Discussion}

The dynamics of changes in the studied indicators was unidirectional in all groups. The index of the maximum possible reduction reached a minimum value 24 hours after exercise and gradually returned to the initial values in 72 hours (Figure 1).

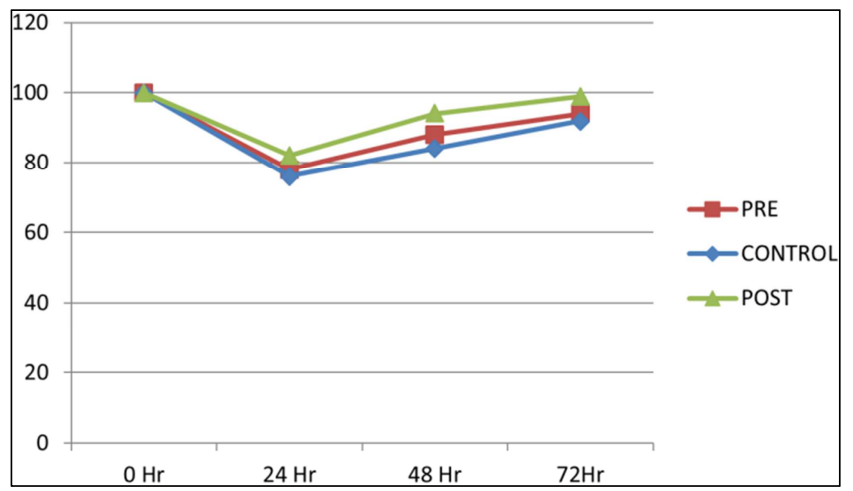

Figure 1. Dynamics of the maximum possible reduction,\% of the initial value.

Between the first group and the control group no significant differences were found in any of the stages of restitution. At the same time, there was a significant difference in recovery in the participants of the second group and the control group at 48 hours after the load $(94 \pm 3.81$ and $84 \pm 3.12$, respectively, $\mathrm{P}<0.05)$, which indicates a faster recovery of contractile ability of the muscles in the studied persons of second group.

When studying the severity of muscle soreness, it was found that its peak occurred at an interval of 48 hours after the load in all groups (Figure 2).

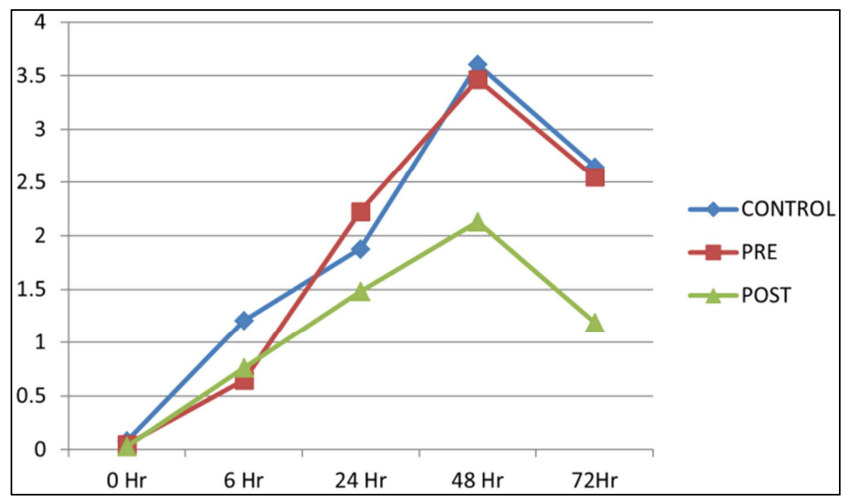

Figure 2. Change in the subjective degree of muscle soreness, $\mathrm{cu}$.

This data coincide with the data of other researchers who determined that the peak of pain is observed 48 hours after the load [11, 21].

Just as in the study of MPR dynamics, there were no significant differences between the indices of participants in the first and control groups. In the case of participants in the third group, significant differences in indices are determined in the intervals 48 and 72 hours after the load.

The dynamics of the change in the concentration of $\mathrm{CK}$ is presented in Figure 3. 


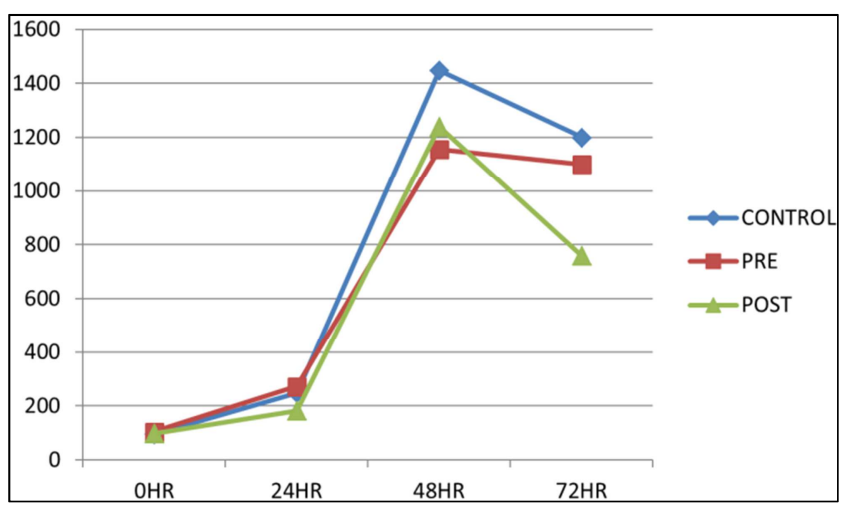

Figure 3. Dynamics of creatine kinase concentration, $U \backslash l$.

It can be seen that its peak values are recorded 48 hours after the load and there are no significant differences between the groups. 72 hours after the load, there are no significant differences between the parameters of the first group and the control also. But when comparing the values of the second group and control parameters in the same time period, significant differences were found (1448 \pm 208 and $864 \pm 113$, respectively, $\mathrm{P}<0.05)$. Creatine kinase, a common marker of muscle damage, is most indicative of damage or rupture of the sarcolemma, leading to "leakage" of cytosolic enzymes from the cell to the blood [13]. The cell membrane is also likely to undergo some degree of lipolysis as a result of an imbalance in calcium homeostasis associated with physical exertion. The reaction of the muscle to damage during exercise is two-phase. The first phase, caused by direct mechanical stress, manifests an inflammatory response within a few hours after exercise [17]. The next phase is characterized by an increase in the demand for polypeptides required for muscle recreation [9, 22]. Therefore, it can be concluded that the intake of protein supplements does not reduce muscle damage during excessive physical exertion, but it contributes to faster muscle relaxation through the acceleration of protein synthesis.

\section{Conclusion}

It has been established that the intake of protein supplements immediately after physical exertion reduces the duration of the subjective sensation of muscle soreness and promotes the fastest regeneration of damaged muscle fibers. This makes it possible to recommend the use of protein supplements directly after physical exertion to optimize the rehabilitation process in the exercise system.

\section{References}

[1] Kurashvili V. Muscle aches and methods of adaptation $\backslash$ Herald of sports innovations - 2014.-49 (49)-P. 7-12.

[2] Medical laboratory technologies: Manual on clinical laboratory diagnostics in 2 volumes. Volume 2\Ed. A. I. Karpischenkol M.: Geotar-Media, 2013.- 792 p.
[3] Beck, Kathryn L.; Thomson, Jasmine S.; Swift, Richard J. Role of nutrition in performance enhancement and postexercise recovery//Open access journal of sports medicine. - 2015. - V. 6. - P. 259-267.

[4] Bianchi G, Marzocchi R, Agostini F, Marchesini G: Update on nutritional supplementation with branched-chain amino acids. Curr Opin Clin Nutr Metab Care. 2005, 8: 83-87.

[5] Breen L, Philp A, Witard OC, Jackman SR, Selby A, Smith K, Baar K, Tipton KD. The influence of carbohydrate-protein coingestion following endurance exercise on myofibrillar and mitochondrial protein synthesis. \I J Physiol.- 2011, -V. 589.-P. 4011-4025.

[6] Dannecker EA, Koltyn KF. Pain During and Within Hours After Exercise in Healthy Adults \Sports Medicine.-2014.-V. 44.-N7.-P. 921-942.

[7] da Luz CR, Nicastro H, Zanchi NE, Chaves DF, Lancha AH: Potential therapeutic effects of branched-chain amino acids supplementation on resistance exercise-based muscle damage in humans. J Int Soc Sports Nutr. 2011, 8: 23-27.

[8] D'Lugos A. C., Luden N. D., Faller J. M., Akers J. D., McKenzie A. I., Saunders M. J. Supplemental Protein during Heavy Cycling Training and Recovery Impacts Skeletal Muscle and Heart Rate Responses but Not Performance // Nutrients. -- 2016. -- Sep. -- V. 8, № 9.

[9] Goodall S, Howatson G: The effects of multiple cold water immersions on indices of muscle damage. Journal of Sports Science and Medicine. 2008, 7: 235-241.

[10] Jackman SR, Witard OC, Jeukendrup AE, Tipton KD: Branched-chain amino acid ingestion can ameliorate soreness from eccentric exercise. Med Sci Sports Exerc. 2010, 42: 962970.

[11] Hansen M., Bangsbo J., Jensen J., Bibby B. M., Madsen K. Effect of Whey Protein Hydrolysate on Performance and Recovery of Top-Class Orienteering Runners // International Journal of Sport Nutrition and Exercise Metabolism. -- 2015. - Apr. -- V. 25, № 2. -- P. 97-109.

[12] Hoffman J, Ratamess N., Tranchina C., Rashti S., Kang J, Faigenbaum A. Effect of Protein-Supplement Timing on Strength, Power, and Body-Composition Changes in Resistance-Trained Men\\ International Journal of Sport Nutrition and Exercise Metabolism.-2009.-V. 19.-N2.-P. 172185 .

[13] Howatson G, Hough P, Pattison J, Hill JA, Blagrove R, Glaister M, Thompson KG: Trekking poles reduce exerciseinduced muscle injury during mountain walking. Med Sci Sports Exerc. 2010, 43: 140-145.

[14] Howatson G, van Someren KA: Evidence of a contralateral repeated bout effect after maximal eccentric contractions. Eur J Appl Physiol. 2007, 101: 207-214.

[15] Howatson G, McHugh MP, Hill JA, Brouner J, Jewell AP, van Someren KA, Shave RE, Howatson SA: Influence of tart cherry juice on indices of recovery following marathon running. Scand J Med Sci Sports. 2010, 20: 843-852.

[16] Howatson G, van Someren KA: The prevention and treatment of exercise-induced muscle damage. Sports Med. 2008, 38: 483-503. 
[17] Jager R., Kerksick C. M., Campbell B. I., Cribb P. J., Wells S. D., Skwiat T. M., Purpura M., Ziegenfuss T. N., Ferrando A. A., Arent S. M., Smith-Ryan A. E., Stout J. R., Arciero P. J., Ormsbee M. J., Taylor L. W., Wilborn C. D., Kalman D. S., Kreider R. B., Willoughby D. S., Hoffman J. R., Krzykowski J. L., Antonio J. International Society of Sports Nutrition Position Stand: protein and exercise // Journal of the International Society of Sports Nutrition. -- 2017. -- Jun. -- T. 14.

[18] Kephart W. C., Mumford P. W., McCloskey A. E., Holland A. M., Shake J. J., Mobley C. B., Jagodinsky A. E., Weimar W. H., Oliver G. D., Young K. C., Moon J. R., Roberts M. D. Post-exercise branched chain amino acid supplementation does not affect recovery markers following three consecutive high intensity resistance training bouts compared to carbohydrate supplementation // Journal of the International Society of Sports Nutrition. -- 2016. -- Jul. -- V. 13.

[19] McHugh MP: Recent advances in the understanding of the repeated bout effect: the protective effect against muscle damage from a single bout of eccentric exercise. Scand J Med Sci Sports. 2003, 13: 88-97.

[20] Matsumoto K, Koba T, Hamada K, Sakurai M, Higuchi T, Miyata H: Branched-chain amino acid supplementation attenuates muscle soreness, muscle damage and inflammation during an intensive training program. J Sports Med Phys Fitness. 2009, 49: 424-431.

[21] Nosaka K, Sacco P, Mawatari K: Effects of amino acid supplementation on muscle soreness and damage. Int J Sport Nutr Exerc Metab. 2006, 16: 620-635.

[22] Ren G. X., Yi S. Q., Zhang H. R., Wang J. Ingestion of soy- whey blended protein augments sports performance and ameliorates exercise-induced fatigue in a rat exercise model // Food \& Function. -- 2017. -- Feb. -- V. 8, № 2. -- P. 670-679.

[23] Sharp CP, Pearson DR: Amino acid supplements and recovery from high-intensity resistance training. J Strength Cond Res. 2010, 24: 1125-1130.

[24] Shimomura Y, Kobayashi H, Mawatari K, Akita K, Inaguma A, Watanabe S, Bajotto G, Sato J: Effects of squat exercise and branched-chain amino acid supplementation on plasma free amino acid concentrations in young women. J Nutr Sci Vitaminol. 2009, 55: 288-291.

[25] Schoenfeld B. J., Contreras B. Is Postexercise Muscle Soreness a Valid Indicator of Muscular Adaptations?\\ Strength and Conditioning Journal.-2013.-V. 35.-N5-P. 16-21.

[26] Stock MS, Young JC, Golding LA, Kruskall LJ, Tandy RD, Conway-Klaassen JM, Beck TW: The effects of adding leucine to pre and postexercise carbohydrate beverages on acute muscle recovery from resistance training. J Strength Cond Res. 2010, 24: 2211-2219.

[27] Wolfe A. S., Brandt S. A., Krause I. A., Mavison R. W., Aponte J. A., Ferguson-Stegall L. M. Shorter Duration Time Trial Performance and Recovery Is Not Improved by Inclusion of Protein in a Multiple Carbohydrate Supplement // Journal of Strength and Conditioning Research. -- 2017. -- Sep. -- V. 31, № 9. -- P. 2509-2518.

[28] Yuan J., Jiang B., Li K., Shen W., Tang J. L. Beneficial Effects of Protein Hydrolysates in Exercise and Sports Nutrition // Journal of Biological Regulators and Homeostatic Agents. -2017. -- Jan-Mar. -- T. 31, № 1. -- C. 183-188. 